\title{
Cytogenetic characterization of Taphronota thaelephora Stal. 1873. (Orthoptera: Pyrgomorphidae) from Cameroon. II. Description of mitotic chromosomes
}

\author{
Richard Akwanjoh SEINO ${ }^{1 *}$, Ingrid Dongmo TONLEU ${ }^{1}$ and Yacouba MANJELI ${ }^{2}$ \\ ${ }^{I}$ Department of Animal Biology, Faculty of Science, University of Dschang, Cameroon. \\ ${ }^{2}$ Department of Animal Production, Faculty of Agriculture and Agronomic Sciences (FASA), \\ University of Dschang, Cameroon. \\ "Corresponding author, E-mail: raseino@yahoo.co.uk; Tel.: +237-77 311434.
}

\begin{abstract}
Taphronota thaelephora Stal. 1873 is generating a lot of interest for biological and cytogenetic studies because it has demonstrated the potential for a future veritable pest in Cameroon. The lack of previously established karyotype for the species has necessitated this study. Testes from five adult individuals collected from Balepa - Mbouda in the West Region of Cameroon and treated with $1 \%$ colchicine were analyzed using the Lactic-Propionic Orcein squash technique. Mitotic metaphase chromosomes were measured directly from the microscope and pictures taken with the help of the Lietz photomicroscope. Pictures were processed with the Microsoft Office Picture Manager. The species revealed the standard Pyrgomorphidae karyotype of $2 \mathrm{n} \delta=19$ acrocentric chromosomes. Mean length of the chromosomes ranged from $8.10 \pm 1.7$ to $3.75 \pm 0.0 \mu \mathrm{m}$. Analysis of Relative Chromosome Lengths (RCL) revealed three distinct size groups of long, medium and short. The karyotype of T. thaelephora was made up of 2 pairs of long chromosomes (2LL) and the X-chromosome, 6 pairs of medium chromosomes (6MM) and 1 pair of short chromosomes (1SS). The mean lengths of the long, medium and short sized chromosomes were $7.2 \mu \mathrm{m}, 4.8 \mu \mathrm{m}$ and $3.51 \mu \mathrm{m}$ respectively. The $\mathrm{X}$-chromosome was the shortest of the large chromosomes with a mean length of $6.75 \mu \mathrm{m}$.
\end{abstract}

(C) 2012 International Formulae Group. All rights reserved.

Keywords: Karyotype, Chromosome length, Relative Chromosome Length (RCL), Chromosome size groups, Taphronota thaelephora.

\section{INTRODUCTION}

Taphronota thaelephora Stal. is a common grasshopper in the West Region of Cameroon. It is a known pest of a wide variety of crops (Nonvieller, 1984). Seino et al. (2001) observed that the species prefers the leaves of crops like Venonia amydalina (Bitter leaf), Manihot esculenta (Cassava),
Ipomea batatas (Sweet potato), Solanium tuberosum (Irish potato), Solanum nigrum (Huckle berry), Amaranthus hybridus (green), and Thea sinensis (Tea) commonly cultivated in the West Region of Cameroon. Two distinct populations of $T$. thaelephora are known in this region. They include the dry 
populations that is encountered in the field during the months of November to February and the wet season populations encountered in the field during the months of April to June (Nembot, 1999; Njukeng, 2000). T. thaelephora is generating a lot of interest for biological and cytogenetic studies particularly as it has demonstrated the potential for a future veritable pest in Cameroon.

Taphronota thaelephora (Figure 1) is a medium - sized grasshopper. Males measure between 20 to $30 \mathrm{~mm}$ while females measure from 30 to $40 \mathrm{~mm}$. The species is generally green in colour. The head is straight and generally pink in colour. The antennae are black with a yellow band occupying the two terminal segments of the antennae. The pronotum bears a saddle-like dorsal surface or shield, which is rough with several projections. The legs of this species are adapted for jumping. The pro-and mesothoracic legs are generally green while the meta-thoracic legs are pink giving way to a green colour at the femur - tibial junction. The tibia is green while the tarsus is pink in colour. The abdomen is green and is covered by a pair of totally green elytra. The elytra are long and cover the whole abdomen in both male and female.

Seino et al. (2002) investigated meiosis in $T$. thaelephora and reported a karyotype of $2 n=19$ acrocentric chromosomes with XO $\mathrm{XX}$ sex determining mechanism in the species. The meiotic process in this species was normal and chiasmate with a mean chiasma frequency of $14.10 \pm 1.0$ per cell. The $\mathrm{X}$ - chromosome in the species exhibited the reverse type of heteropycnosis in which it was positively heteropycnotic in first meiotic Prophases and negatively heteropycnotic in first meiotic Metaphases and Anaphases. Seino et al. (2007) reported the presence of a single B- chromosome in some individuals of the dry season population of this species collected from Mbouda in the West Region of Cameroon. This chromosome was small, acrocentric and stained to the same intensity as the autosomes throughout meiosis. The presence of this supernumerary chromosome did not affect chiasma frequency in the species.

In continuation with our research on $T$. thaelephora (Seino et al., 2002), we here present a pioneer description of the karyotype of the species based on mitotic chromosomes.

\section{MATERIALS AND METHODS}

In May 2010, five adult individuals of T. thaelephora were collected from the village of Balepa in Mbouda, West Region of Cameroon and used for this study. On capture, the insects were injected with $1 \mathrm{ml}$ of $1 \%$ colchicines and allowed for 24 hours before being killed with chloroform fumes and then dissected in insect saline $(0.68 \%$ $\mathrm{NaCl}$ ) for the testes. The testes obtained were placed in 3:1 ethanol: acetic acid fixative and stored in the refrigerator at $4{ }^{\circ} \mathrm{C}$ until used.

\section{Chromosome preparation}

Chromosome smears were prepared using the Lactic-Propionic-Orcein squash technique (Seino et al., 2010). Two to three testicular follicles were placed on a clean microscope glass slide. They were first flooded with $45 \%$ acetic acid for five minutes. This made the cells to swell. After blotting off the acid, the tissue was next flooded with one or two drops of Lactic-Propionic-Orcein stain and macerated using the sharp pointed end of a dissecting needle. This permitted the stain to penetrate into the tissue. The preparations were then incubated at room temperature between ten and fifteen minutes while making sure that the stain did not dry off. A cover slide was next placed on the tissue, held in place with the thumb and forefinger before gently tapping with the wooden end of a dissecting needle. This enabled the cells to disperse and force out excess stain. The preparation was then wrapped in filter paper and squashed between the thumb and the top of the laboratory table. The filter paper absorbed excess stain. The edges of the cover slide were sealed with colourless nail vanish to temporarily preserve the preparation. 


\section{Microscopical examination and photography}

The chromosome smears thus prepared were examined using the Fisher laboratory microscope. Slides were initially scanned under a 10X objective and nuclei of interest further examined under a high power objective 40X.

Chromosome morphology was determined by examining the shapes of chromosome in meiotic Anaphase-I, Metaphase-II and Anaphase-II and classified as per the criteria of Williams and Ogunbiyi (1995).

Photographs of mitotic chromosome smears were taken with the Lietz digital photomicroscope using the oil immersion lens, 100X. Sufficient photographs were taken and mitotic metaphases were processed using the Microsoft Office Picture Manager.

\section{Measurements and calculations}

The lengths of individual chromosomes were measured directly from the microscope using ocular and stage micrometers. Each chromosome per mitotic Metaphase cell was measured several times by each of the authors until the measurement obtained did not vary. The ocular divisions obtained were then recorded and converted into micrometers upon calibration with the stage micrometer. Individual chromosome pairs were identified on the basis of length (Cody and Jeffrey, 2006).

Relative chromosome length (RCL) is the length of each chromosome expressed as a percentage of the total haploid autosome length in the nucleus (Paris Conference, 1971). This was calculated by adding together the lengths of all the autosomes in one nucleus, then dividing by 2 because they are paired, to obtain the total haploid length. Then each chromosome length was divided by the total haploid length and multiplied by 100 to gain a percentage result.

Data on RCL were used to construct graphs to determine size groups. To confirm the size groups of long, medium and short obtained, mean chromosome lengths were subjected to the Duncan's Multiple Range test, (DMRT) and tested at the 5\% level of significance (Clewer and Scarisbrick, 2001).

\section{RESULTS}

\section{Chromosome number}

The chromosomal analysis revealed a consistent modal chromosome number of 19 (Figure 2). Meiotic analysis was also undertaken in all the individuals examined. First meiotic Diplotene and Metaphases showed 9 evenly paired bivalents and a presumed univalent X-chromosome (Figure 3 $\& 4)$. This lent support to the interpretation of the mitotic smears having 19 chromosomes. A diploid number of $2 n \quad \hat{\sigma}=19$ was however expected since Seino et al. (2002) had reported a similar chromosome count for the species. Moreover, species in the family Pyrgomorphidae are known to be characterized by a diploid number of $2 \mathrm{n} \widehat{\delta}=$ 19 (White, 1973; Hewitt, 1979).

\section{Chromosome morphology}

The chromosomes in the species were rod-shaped with sister chromatids separated gradually from a tapered end towards the other end. The two chromatids in each chromosome were not coiled around each other and short arms were not distinct in the chromosomes (Figure 2-7). Centromeres in the chromosomes were inferred to be in the tapered terminal regions where the sister chromatids were in close contact. On this basis the chromosomes in T. thaelephora were deemed to be acrocentric or telocentric in morphology. Examination of meiotic chromosomes in first meiotic Anaphases (Figure 5) and second meiotic Metaphases and Anaphases (Figure $6 \& 7$ ) lent support to the interpretation that chromosomes in $T$. thaelephora are acrocentric in morphology. The chromosomes in first meiotic Anaphases were each made up of two chromatids held together at one end by the centromere and repulsion between sister chromatids had taken place distal to this centromere. This conferred 
a V-shaped appearance on the chromosomes (Figure 6). This V- shaped configuration is what is expected for telo-acrocentric chromosomes in Anaphase-1. Teloacrocentric chromosomes in Anaphase -1 would have two arms with the centromere at the point of convergence. In second meiotic Metaphases, the chromosomes were made up of two chromatids held together at one end by the centromere and distal to this centromere, there was repulsion between the sister chromatids conferring a V-shaped appearance on the chromosomes. However, in this case, the chromosomes were grouped in a circular manner at the two poles of the cell (Figure 6). Chromosomes in second meiotic Anaphases were clearly single stranded and appeared Ior rod-shaped (Figure 7). They were therefore not folded. According to the description of Williams and Ogunbiyi (1995), the chromosomes in $T$. thaelephora can be said to be acrocentric in morphology.

\section{Chromosome lengths}

The lengths, Relative Chromosome Lengths (RCL) and morphology of the chromosomes in T. thaelephora are shown in Table 1. This table revealed that in $T$. thaelephora, the length of the total haploid (n) set was $54.46 \mu \mathrm{m}$. The lengths of the chromosomes ranged from 8.10 to $3.51 \mu \mathrm{m}$. There was a gradual decrease in chromosome length from the largest to the smallest chromosome.

The RCLs in Table 1 were used to construct the graphs in Fig. 8 in order to determine if the chromosomes occurred in different size groups. The graphs revealed that the chromosomes in the karyotype of $T$. thaelephora occurred in three size groups of long, medium and short. They included 2 pairs of long, 6 pairs of medium and 1pair of short autosomes (2LL, 6MM, 1SS). This distribution was supported by the karyotype constructed for the species shown in Figure 9.

The lengths of the long chromosomes ranged from 8.10 to $6.75 \mu \mathrm{m}$, the medium chromosomes ranged from 5.13 to $4.55 \mu \mathrm{m}$ while the length of the short chromosome was $3.51 \mu \mathrm{m}$. The X-chromosome in this species had a mean length of $6.75 \mu \mathrm{m}$ and was therefore the shortest of the long chromosomes in $T$. thaelephora. The mean lengths of the long, medium and short autosomal pairs were $7.23 \mu \mathrm{m}, 4.80 \mu \mathrm{m}$ and $3.51 \mu \mathrm{m}$ respectively.

The mean chromosome lengths (Table 1) were next compared using the Duncan Multiple Range Test (DMRT) to determine if they were significantly different for the long, medium and short size groups. The results of DMRT revealed that the mean length of the long chromosomes was significantly higher $(\mathrm{P}<0.05)$ than that of the medium chromosomes and that the mean length of the medium chromosomes was significantly higher $(\mathrm{P}<0.05)$ than that of the short chromosomes. This helped us to confirm that the chromosomes in the karyotype of $T$. thaelephora occurred in three distinct size groups of long, medium and short.

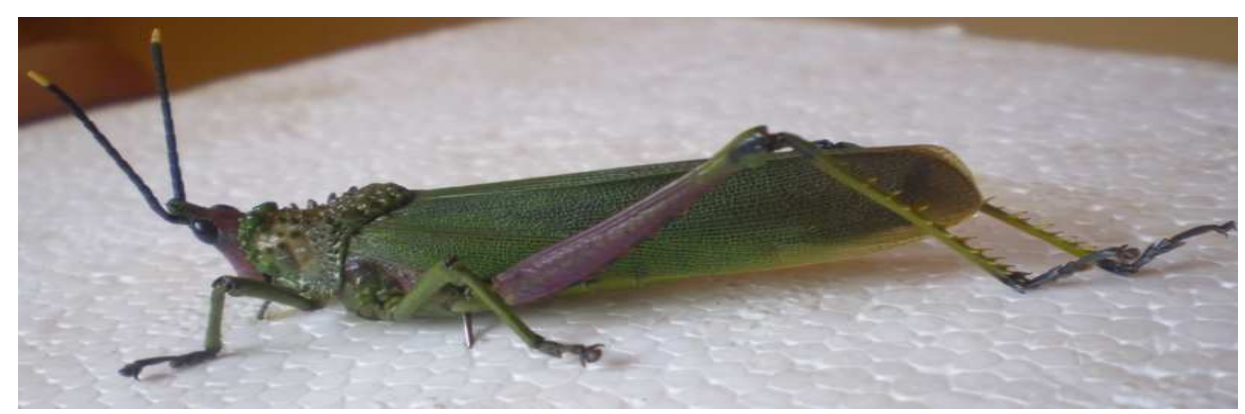

Figure 1: Picture of male T. thaelephora. 


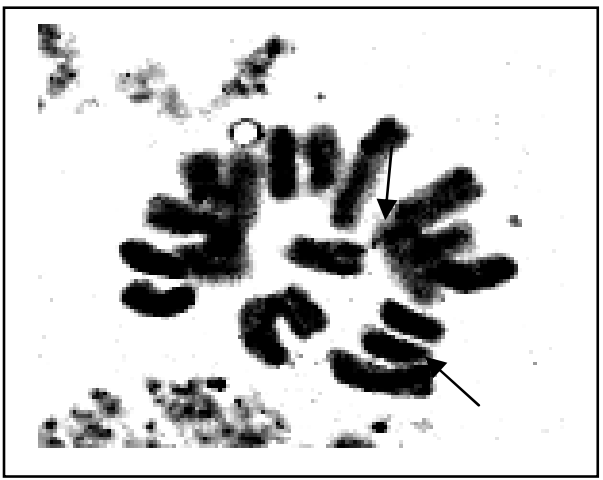

Figure 2: Mitotic Metaphase chromosomes in T. thaelephora.

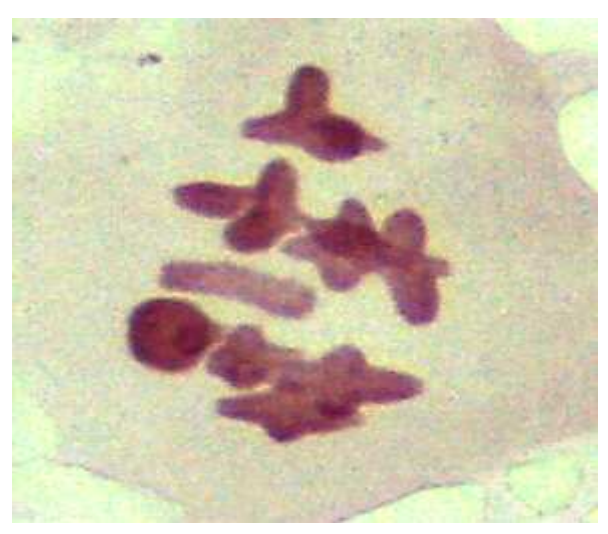

Figure 4: First meiotic Metaphase in T. thaelephora.

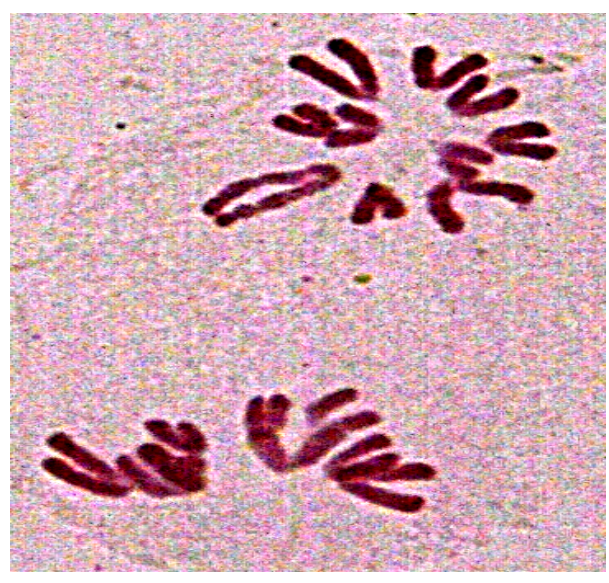

Figure 6: Second meiotic Metaphase in T. thaelephora.

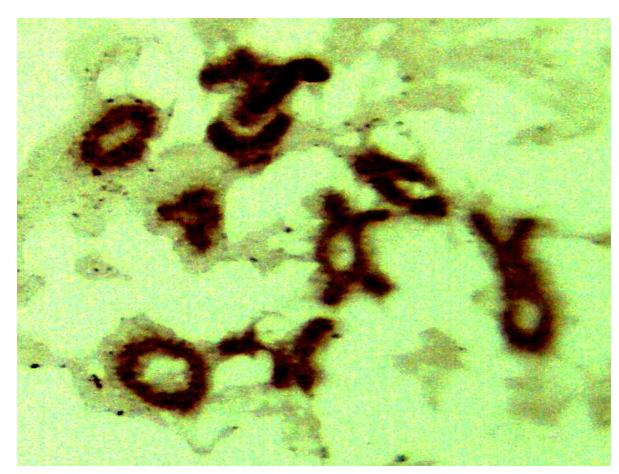

Figure 3: Diplotene in T. thaelephora.

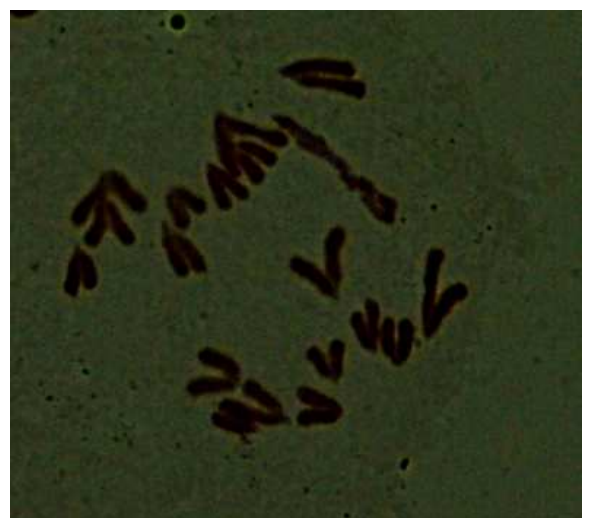

Figure 5: First meiotic Anaphase in $T$. thaelephora.

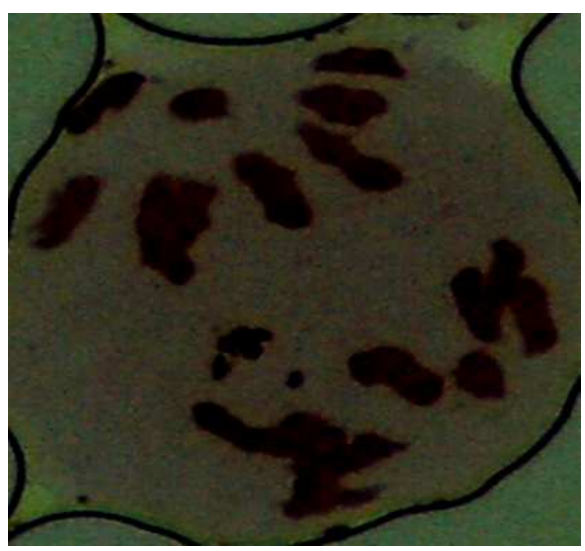

Figure 7: Second meiotic Anaphase in T. thaelephora. 


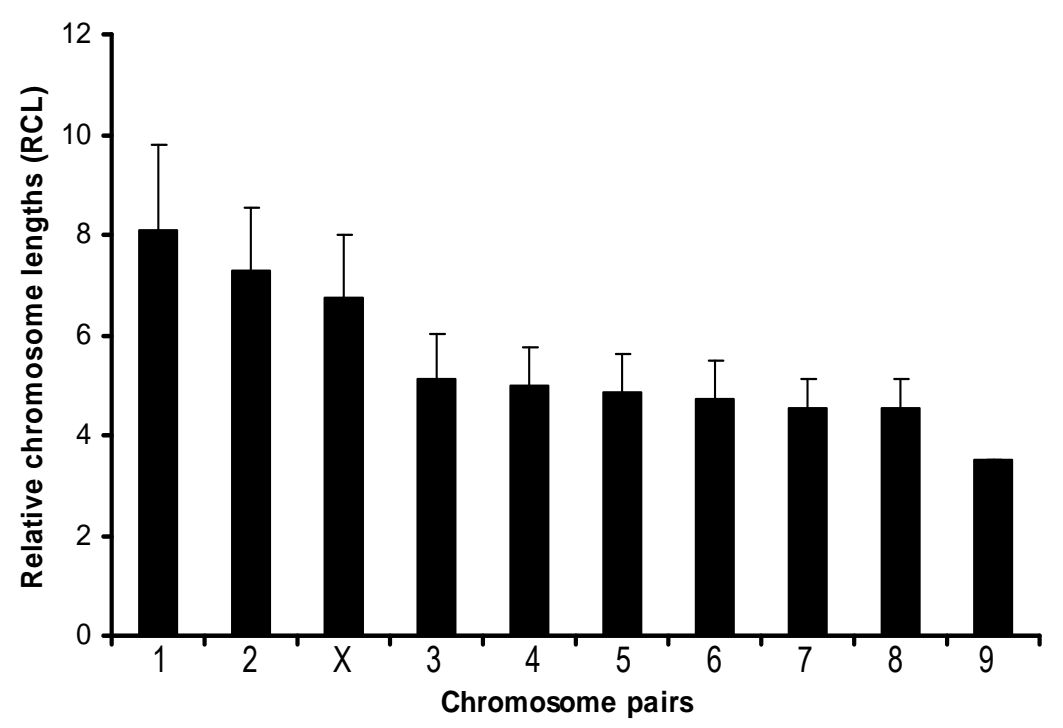

Figure 8: Relative Chromosome Length (RCL) by chromosome pair in T. thaelephora.

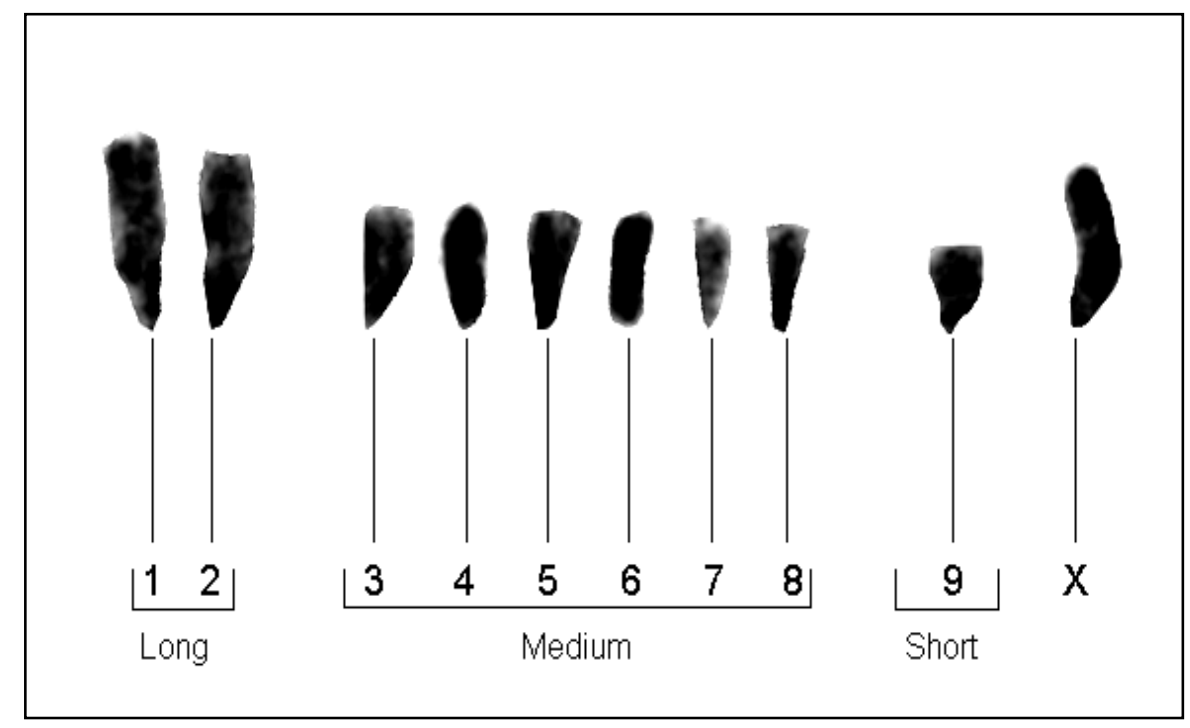

Figure 9: Karyotype of T. thaelephora. Chromosomes occur in size groups of long medium and short. 
R. A. SEINO et al. / Int. J. Biol. Chem. Sci. 6(4): 1624-1632, 2012

Table 1: Chromosome lengths $(\mu \mathrm{m}), \mathrm{RCL}$, and chromosome size groups in Taphronota thaelephora.

\begin{tabular}{|c|c|c|c|c|c|c|c|c|c|c|}
\hline & \multicolumn{10}{|c|}{ Chromosome pairs } \\
\hline & 1 & 2 & $\mathbf{X}$ & 3 & 4 & 5 & 6 & 7 & 8 & 9 \\
\hline $\begin{array}{l}\text { Chromosome } \\
\text { length }(\mu \mathrm{m})\end{array}$ & $8.10 \pm 1.70$ & $7.29 \pm 1.27$ & $6.75 \pm 1.27$ & $5.13 \pm 0.91$ & $4.99 \pm 0.78$ & $4.86 \pm 0.74$ & $4.73 \pm 0.74$ & $4.55 \pm 0.57$ & $4.55 \pm 0.57$ & $3.51 \pm 0.00$ \\
\hline RCL (\% of n- & 14.87 & 13.39 & 12.40 & 9.42 & 9.16 & 8.92 & 8.69 & 8.36 & 8.36 & 6.45 \\
\hline $\begin{array}{l}\text { set) } \\
\text { Chromosome } \\
\text { size groups }\end{array}$ & & LARGE & & & & ME & UM & & & SMALL \\
\hline $\begin{array}{l}\text { Mean lengths } \\
(\mu \mathrm{m})\end{array}$ & & 7.23 & & & & & & & & 3.51 \\
\hline
\end{tabular}




\section{DISCUSSION}

The karyotype described in this paper is an improvement over the first report of Seino et al. (2002) in which chromosomes were simply counted and their morphology (position of the centromere) described. In their study, Seino et al. (2002) did not also measure the lengths of the chromosomes and hence determine the size groups in which the chromosomes occurred. Therefore the lack of previously established karyotype for $T$. thaelephora has necessitated this decision as to the chromosomal arrangements in Figures 2 and 8. During this study, the measurements of chromosome lengths have made it possible to match homologues on the basis of length, analyse statistically the data obtained and hence group the chromosomes according to size.

This study confirmed $2 \mathrm{n}=19$ as the characteristic diploid chromosome number for males in this species which according to White (1973) is believed to be initial for the family Pyrgomorphidae. The chromosomes in T. thaelephora are also certainly acrocentric in morphology even though short arms were not visible. The non-visibility of short arms in these chromosomes could be explained by the fact that the chromosome preparations were not the best. However, the vast majority of Pyrgomorphidae possess a fundamental complement of $2 \mathrm{n}=19(\mathrm{XO})$ acrocentric chromosomes (Hewitt, 1979; Mesa and Fontanetti, 1983; Santos et al., 1983).

The chromosomes in $T$. thaelephora ranged in length from $8.10 \pm$ to $3.51 \pm 00.00$ $\mu \mathrm{m}$ and were comparable to the chromosomes in Z. variegatus (Pyrgomorphidae) that ranged in length from 8.20 to $2.51 \mu \mathrm{m}$ (Faluyi and Olorode, 1988). The results obtained in this study also revealed that the chromosomes in T. thaelephora could be divided into three groups of long, medium and short a characteristic of Orthoptera grasshoppers (Warchalowska-Sliva et al., 2002). Accordingly, the karyotype of $T$. thaelephora was characterised by 2 long pairs, 6 medium pairs and 1 short pair of autosomes. Since this karyotype was from male individuals, it seems likely that the unpaired chromosome represents the $\mathrm{X}$ - or sex chromosome. This $\mathrm{X}$-chromosomes in $T$. thaelephora measured $6.75 \mu \mathrm{m}$ and belonged to the group of long chromosomes. Faluyi and Olorode (1988) obtained a comparable length for the Xchromosome in $Z$. variegatus (Pyrgomorphidae) that measured $6.77 \mu \mathrm{m}$.

These results lend support to the characteristic Orthoptera karyotypes with chromosomes in three size groups of long, medium and short (White, 1973).

\section{REFERENCES}

Clewer AG, Scarisbrick DH. 2001. Practical Statistics and Experimental Design for Plant and Crop Science. John Wiley \& Sons.

Cody RP, Jeffrey KS. 2006. Applied Statistics and the SAS Programming Language (5th edn). Prentice Hall: New Jersey.

Faluyi JO, Olorode O. 1988. Mitotic chromosome studies in the nymphal and adult populations of Zonocerus variegatus L. (Orthoptera: Pyrgomorphidae). Journal of African Zoology, 102(4): 475 - 480.

Hewitt GM. 1979. Grasshoppers and Crickets. Animal cytogenetics. Insecta, I. Orthoptera (vol. 3). Grebruger Borntaeger Ed. : Berlin, Stuttgart.

John B, King M. 1983. Population cytogenetics of Atractomorpha similis. I. C-band variation. Chromosoma, 88: 57 68.

Mesa A, Fontanetti CS. 1983. Karyotypes of nine Brazilian species of Acrididae Acridoidea. Brazilian Journal of Genetics, 6(2): 295 - 305.

Nembot E. 1999. Comportement nutritionnel du croquet peste Taphronota thaelephora Stal. (Acridomorphoidea : Pyrgomorphidae). Mémoire de Maîtrise en Biologie Animale, Université de Dschang, Cameroun. 
Nonvieller G. 1984. Catalogue des Insectes du Cameroun d'Intérêt Agricole. PRIFAS: Montpellier.

Paris conference. 1971. Standardization in Human cytogenetics. Birth Defects: Original.

Article Series 8: 7, 1972 New York: The National Foundation (Reprinted in Cytogenetics, 11: 1313 -362 (1972)).

Santos JL, Arana P, Giraldez R. 1983. Chromosome C-banding patterns in Spanish Acridoidea. Genetica, 61: 65 74.

Seino RA, Mpoame M, Nembot E. 2001. Observations on the food preferences of Taphronota thaelephora Stal. (Orthoptera: Pyrgomorphidae). J. Sci. Tech. \& environ., 1: 129 - 134.

Seino RA, Focho DA, Njukeng FA. 2002. Observations on the meiotic process in the African pest grasshopper Taphronota thaelephora Stal. (Orthoptera: Pyrgomorphidae). J. Acad. Sci., 2(1): 36.

Seino RA, Manjeli Y, Focho DA, Shambo DN. 2007. The B chromosome in the meiotic process of the African pest grasshopper Taphronota thaelephora Stal. (Orthoptera: Pyrgomorphidae). Int. J. Biol. Chem. Sci., 1(2): 151 - 157.

Seino RA, Akongnui T. 2010. Meiotic studies of Acrida turrita Linnaeus, 1758), Paracinema luculenta Karsch, 1896 and Morphacris fasciata (Thunberg 1815) (Orthoptera: Acrididae). Int. J. Biol. Chem. Sci., 4(6): 1914 - 1921.

Warchalowska-Sliva E, Kostia D, Sliwa L. 2002. Cytological and morphological differences between two species of the genus Tettigonia (Orthoptera, Tettigonidae) from Korea. Folia Biological (Krakow), 50(1 - 2): 23 - 28.

White MJD. 1973. Animal cytology and Evolution $\left(3^{\text {rd }}\right.$ edn). The Cambridge University Press.

Williams GO, Ogunbiyi BI. 1995. Chromosome morphology and meiosis in Zonocerus variegatus L. (Orthoptera: Pyrgomorphidae). Cytologia, 60: 111116. 